\title{
Combining multivariate genomic approaches to elucidate the comorbidity between ASD and ADHD
}

Hugo Peyre ${ }^{1,2,3,4^{*}}$, Tabea Schoeler ${ }^{1}$, Chaoyu Liu $^{1}$, Camille Michèle Williams $^{2}$, Nicolas Hoertel $^{5,6}$, Alexandra Havdahl ${ }^{7}$, Jean-Baptiste Pingault ${ }^{1,8}$

1. Department of Clinical, Educational and Health Psychology, University College London, London, UK.

2. Laboratoire de Sciences Cognitives et Psycholinguistique (ENS, EHESS, CNRS), Ecole Normale Supérieure, PSL University, Paris, France.

3. Neurodiderot. INSERM UMR 1141, Paris Diderot University, Paris, France.

4. Department of Child and Adolescent Psychiatry, Robert Debré Hospital, APHP, Paris, France.

5. INSERM UMR 894, Psychiatry and Neurosciences Center; Paris Descartes University, PRES Sorbonne Paris Cité, Paris, France.

6. Department of Psychiatry, Corentin Celton Hospital, APHP, Issy-les-Moulineaux; Paris Descartes University, PRES Sorbonne Paris Cité, Paris, France.

7. Nic Waals Institute, Lovisenberg Diaconal Hospital, 0853 Oslo, Norway; Department of Mental Disorders, Norwegian Institute of Public Health, Oslo, Norway; MRC Integrative Epidemiology Unit, University of Bristol, Bristol, UK.

8. Social, Genetic and Developmental Psychiatry Centre, Institute of Psychiatry, Psychology and Neuroscience, King's College London, London, UK

Running title: Genetic of the comorbidity between ASD and ADHD.

*CORRESPONDING AUTHOR: Hugo Peyre, MD, PhD: LSCP, Département d'Etudes Cognitives. Ecole Normale Supérieure, 29 rue d'Ulm, 75005 Paris, France. Email: peyrehugo@yahoo.fr. Phone: 0033(0)144322619. Fax: 0033(0)144322619 
Acknowledgement: JBP is supported by the Medical Research Foundation Emerging Leaders $1^{\text {st }}$ Prize 2018 Adolescent Mental Health.

\section{Conflict of interest}

On behalf of all authors, the corresponding author states that there is no conflict of interest.

\section{Keywords}

Autism Spectrum Disorder; Attention Deficit Hyperactivity Disorder; genetic factors; colocalization; genomic structural equation modelling; GWAS.

\section{Tables \& Figures}

Number of Figures: 1

Number of Tables: 4

Number of Supplementary Figures: 10

Number of Supplementary Tables: 4

Number of supplementary Data: 3 


\section{ABSTRACT}

2 Background: Several lines of evidence point toward the presence of shared genetic factors

3 underlying Autism Spectrum Disorder (ASD) and Attention Deficit Hyperactivity Disorder

4 (ADHD). However, Genome-Wide Association Studies (GWAS) have yet to identify risk

5 variants (i.e. Single-Nucleotide Polymorphisms, SNPs) shared by ADHD and ASD.

6 Methods: Two complementary multivariate analyses - genomic structural equation

7 modelling (SEM) and colocalization analysis - were exploited to identify the shared SNPs for

8 ASD and ADHD, using summary data from two independent GWAS of ASD $(\mathrm{N}=46,350)$

9 and ADHD individuals ( $\mathrm{N}=55,374)$.

10 Results: Genomic SEM identified 7 novel SNPs shared between ASD and ADHD ( $p_{\text {genome- }}$

11 wide $<5 e-8$ ), including three SNPs that were not identified in any of the original univariate

12 GWAS of ASD and ADHD (rs227378, rs2391769 and rs325506). We also mapped 4 novel

13 genes (MANBA, DPYD, INSM1, and PAX1) to SNPs shared by ASD and ADHD, as well as

144 genes that had already been mapped to SNPs identified in either ASD or ADHD GWAS

15 (SORCS3, XRN2, PTBP2 and NKX2-4). All the shared genes between ADHD and ASD

16 were more prominently expressed in the brain than the genes mapped to SNPs specific to

17 ASD or ADHD. Colocalization analyses revealed that $44 \%$ percent of the SNPs associated

18 with ASD $(p<1 \mathrm{e}-6)$ colocalized with ADHD SNPs and $26 \%$ of the SNPs associated with

19 ADHD $(p<1 \mathrm{e}-6)$ colocalized with ASD SNPs.

20 Conclusions: Using multivariate genomic analyses, the present study reveals the shared

21 genetic pathways that underlie ASD and ADHD. Further investigation of these pathways may

22 help identify new targets for treatment of these disorders. 


\section{INTRODUCTION}

27 Attention Deficit Hyperactivity Disorder (ADHD) and Autism Spectrum Disorder (ASD) are

28 two common neurodevelopmental disorders (1) with a prevalence in children between 5 to

$297 \%$ (2) and 1 to $2 \%$, respectively (3). ADHD is characterized by symptoms of inattention,

30 impulsivity, and hyperactivity, and ASD by a deficit in social communication as well as

31 restricted and repetitive patterns of interests and behaviors. Although both disorders are

32 distinctive in terms of diagnostic criteria, there is a considerable overlap in symptomatology.

33 Individuals with ASD commonly display inattention, impulsivity, and hyperactivity

34 symptoms (4) and likewise, individuals with ADHD often have impaired social and

35 communication skills (5-7). In addition, ASD and ADHD are frequently comorbid;

36 approximately one third of children with ASD are also diagnosed with ADHD $(8,9)$ and one

37 fifth of children with ADHD meet diagnostic criteria for ASD (7).

39 Little is known about what causes the association between ASD and ADHD. Both disorders

40 are thought to be caused by a complex interplay between environmental and genetic risk

41 factors $(10,11)$. Shared environmental risk factors, such as preterm birth $(12,13)$ or prenatal

42 exposure to valproate $(14,15)$ might partially explain the association between the disorders.

43 Several lines of evidence also point toward the presence of shared genetic factors in ASD and

44 ADHD. In family-based studies, relatives of children with ASD are at a higher risk for

45 ADHD than relatives of children without ASD $(16,17)$ and in twin studies, researchers report

46 strong genetic correlations between traits related to ADHD and traits related to ASD $(18,19)$.

47 Moreover, a similar burden of rare protein-truncating variants has been found in individuals

48 with ASD and those with ADHD (20). Most of the rare Copy Number Variants (CNVs) that

49 are linked to ASD are also associated with $\operatorname{ADHD}(21,22)$. But less than $10 \%$ of ASD and

50 ADHD liabilities can be accounted for by rare genetic variants $(22,23)$. To this day, Genome- 
51 Wide Association Studies (GWAS) have not yet identified shared common risk variants (i.e.

52 Single-Nucleotide Polymorphisms, SNPs) for ADHD and ASD (10,24,25). This is because

53 the shared genetic risk has not yet been modelled using appropriate multivariate genomic

54 approaches, such as genomic structural equation modelling (Genomic SEM) (26) or

55 colocalization (27). Genomic SEM appears particularly useful to identify novel shared risk

56 SNPs that remained undetected in univariate GWAS of overlapping traits $(26,28)$, while

57 colocalization allows identification of shared and specific SNPs after taking linkage

58 disequilibrium (LD) into account. This latter method already elucidated the shared genetic

59 risk between autoimmune diseases (29), lipid levels and cardiovascular outcomes (30), as

60 well as schizophrenia and gene expression within human brain tissue $(31,32)$.

62 As an alternative possibility, the association between ASD and ADHD might be explained by

63 direct causal relationships between the two phenotypes, unidirectionally or bidirectionally.

64 ADHD might lead to secondary impairments in social interaction and behavioral flexibility

65 (33), while ASD might contribute to secondary attention problems, hyperactive and

66 impulsive behaviours. These directional relationships could bias our interpretation of the

67 colocalization analysis considering that a given SNP may colocalize between ADHD and

68 ASD not because of shared genetic risk but because of the increase of ADHD symptoms in

69 individuals with ASD or the increase of ASD symptoms in individuals with ADHD. To

70 remedy to issue of directionality, the present study will be the first to conduct a Bidirectional

71 Mendelian randomization (MR) analysis on ASD and ADHD GWAS to determine the effect

72 of $\mathrm{ADHD}$ on $\mathrm{ASD}$ and the effect of $\mathrm{ASD}$ on $\mathrm{ADHD}(34,35)$.

74 This study used the aforementioned multivariate genomic approaches (Genomic SEM and 75 colocalization) to identify SNPs shared by ASD and ADHD and SNPs specific to each 
76 disorder. Functional analyses were performed on shared and specific common genetic

77 variants. Finally, a bidirectional Mendelian randomization analysis was performed to explore

78 whether the shared genetic risk between ASD and ADHD was interpretable in terms of

79 (reciprocal) causal relationships.

81 METHODS AND MATERIALS

82 GWAS Summary Statistics

83 Summary statistics for ASD and ADHD were obtained from the European ancestry subgroup

84 of the Psychiatric Genomics Consortium and iPSYCH (PGC + iPSYCH). We used the most

85 recent GWAS data on ASD (18,381 diagnosed ASD cases and 27,969 controls) (24) and

86 ADHD individuals (20,183 diagnosed ADHD cases and 35,191 controls) (25).

\section{Genomic SEM}

89 Genomic SEM (GenomicSEM R package) models the genetic covariance structure of GWAS

90 summary statistics using LD score regression $(\operatorname{LDSC})(26,36)$ to estimate the association of

91 each SNP with the general factor (a latent variable corresponding to the shared variance

92 between the ASD and ADHD GWAS). In the Genomic SEM, factor loadings were fixed to

93 be equal between ASD and ADHD GWAS.

\section{Colocalization Analyses}

96 Colocalization analyses were conducted on each genome-wide significant $(p<5 \mathrm{e}-8)$ LD-

97 independent $(\mathrm{r} 2>0.2$; window of $500 \mathrm{~kb})$ SNP associated with either ASD, ADHD, or the

98 general factor to account for two types of false results when solely analysing individual SNP

99 association parameters (Supp. Figure 1): (i), a SNP associated with a trait A may be falsely

100 associated with the general factor of Trait A and B because a causal SNP of Trait B is in LD 
101 with the SNP of Trait A; (ii), the general factor might miss some SNPs that are in fact

102 associated with both traits. For example, a shared causal SNP strongly associated with Trait

103 A but less strongly associated with Trait B may erroneously not be linked to the general

104 factor.

105 We used the R packages COLOC (37) and Hyprcoloc (38). Both methods implement a

106 Bayesian test for the colocalization of two associations signals in a selected region (250kb

107 around a SNP under study), using the summary statistics of two traits, to calculate five

108 hypotheses: H0 (no association between the SNP and either trait), H1 (SNP association with

109 trait 1 only), H2 (SNP association with trait 2 only), H3 (SNP association with both traits but

110 not colocalizing, i.e. two distinct SNPs in LD); and H4 (SNP association with both traits and

111 colocalizing). We set the prior probability that a SNP is causal in each trait to be identical

112 (1e-4 is the recommended threshold for colocalization analysis in the context of GWAS

$113(27,37,39))$ and the prior probability that a SNP is causal for both traits at 5e-6 (meaning that

1145 out of 100 SNPs that are associated with one trait are also associated with the other). A

115 given SNP was considered to colocalize if $\mathrm{H} 4>0.5$ using COLOC or Hyprcoloc packages

116 (31,40). To estimate the percentage of SNPs that colocalized between both traits more

117 precisely, we performed a similar analysis but at the $p$-value threshold of 1e-6.

118 SNPs colocalizing between ADHD and ASD and associated with the general factor at $\mathrm{p}<5 \mathrm{e}-8$

119 were considered as SNPs shared by ASD and ADHD. Among the SNPs not colocalizing

120 between ADHD and ASD, those associated with a $p$-value below 5e-8 with ASD were

121 classified as SNPs specific to ASD and those associated with a p-value below 5e-8 with

122 ADHD were classified as SNPs specific to ADHD.

\section{Functional Analysis}


125 Functional annotation and analyses were performed separately on SNPs shared by ASD and

126 ADHD (i.e. SNPs colocalizing between ADHD and ASD), and SNPs specific to each

127 disorder using FUMA (Functional Mapping and Annotation of Genome-Wide Association

128 Studies) (http://fuma.ctglab.nl/) (41). Using FUMA SNP2GENE, SNPs are mapped to genes

129 based on physical position (positional mapping of deleterious coding SNPs (CADD

130 score $\geq 12.37$ ) and eQTL associations (we used 13 brain tissue types from the GTEx.v8

131 project). Moreover, SNPs are mapped to genes based on 3D chromatin interactions

132 (chromatin interaction mapping; Hi-C data of 2 tissue types: adult and foetal cortex (42)).

133 Chromatin interaction mapping can involve long-range interactions. GENE 2FUNC then

134 annotates these genes in a biological context. Among the other functions of GENE 2FUNC,

135 tissue specific expression patterns (based on GTEx v6 RNA-seq data) for each gene were

136 visualized as an interactive heatmap and globally for SNPs that are shared by ASD and

$137 \mathrm{ADHD}$ and for those that are specific to ASD and ADHD.

139 Bidirectional Mendelian Randomization Analyses

140 We ran bidirectional summary data Mendelian randomization (MR) analysis to determine the

141 effect of the liability for ADHD on ASD and the liability for ASD on ADHD $(34,35,43)$. LD-

142 independent SNPs (r2>0.001; window of $500 \mathrm{~kb})$ associated with ASD $\left(\mathrm{n}_{\mathrm{SNPs}}=15\right.$, with $\mathrm{p}<1 \mathrm{e}$ -

143 6) were selected as instruments for ASD to estimate the effect of ASD on ADHD.

144 Associations were also ascertained in the opposite direction, using $n=41$ SNPs $(p<1 \mathrm{e}-6)$ as

145 instruments for ADHD. We estimated the main effects using the inverse weighted variance

146 (IVW) estimator, which consists of a linear regression of the instrument-outcome association

147 estimates on the instrument-exposure association estimates, weighted by the inverse of the

148 variance of the instrument-outcome association estimates. A number of MR methods were

149 also employed for sensitivity analyses to examine potential violations of the MR 
150 assumptions, such as exclusion restriction assumption due to pleiotropy (i.e. the SNP used as

151 instrument does not affect directly both exposure and outcome), including the weighted

152 median-based and mode-based because they allow some genetic instruments (i.e. SNPs) to be

153 invalid (44). We also conducted leave-one-out sensitivity analyses to check for a

154 disproportionate influence of individual SNPs on overall effect estimates using the IVW

155 method. We additionally applied MR-Egger regression $(45,46)$ to estimate the intercept

156 (corresponding to a test of directional pleiotropy) in addition to the slope in the regression.

157 The $\mathrm{I}_{\mathrm{GX}}$ statistic was used to test for heterogeneity in MR Egger regression.

158 Because only few SNPs associated with ASD and ADHD at genome-wide significance level

$159(p<5 \mathrm{e}-8)$ are available in the current GWAS (Grove et al. (24), Demontis et al. (25)), MR

160 analyses were conducted at a $p$-value threshold of 1e-6. As MR analysis were performed with

161 instruments below the conventional GWAS threshold (i.e. $p<5$ e-8) we also applied the

162 Mendelian randomization robust adjusted profile score (MR-RAPS) to account for weak

163 instrument bias (47).

\section{RESULTS}

\section{Genomic SEM}

167 Genomic SEM was conducted on 6,971,687 SNPs that were present in both ASD (number of 168 available SNPs=7,757,027) and ADHD (number of available SNPs=8,094,094) GWAS.

169 There were 232 genome-wide significant $(p<5 \mathrm{e}-8)$ SNPs identified in the general factor 170 GWAS, including 7 LD-independent SNPs following clumping. Two of the 7 SNPs 171 (rs1222063 and rs4916723) were previously reported in the ADHD GWAS from which we 172 obtained the summary statistics (Table 1). The Manhattan plot of the general factor GWAS is 173 depicted in Figure 1. 


\section{Colocalization Analyses}

176 The main results of the colocalization analysis are summarized in Table 1. One out of the

177 two SNPs associated with ASD colocalized with ADHD, 4 out of 18 SNPs associated with

178 ASD colocalized with ADHD, and all the 7 SNPs associated with the general factor

179 colocalized between ASD and ADHD GWAS.

180 After clumping, we found two SNPs associated with ASD (and 16 when $p$-value threshold

181 was set at 1e-6) and 18 SNPs associated with ADHD (and 50 when $p$-value threshold was set

182 at 1e-6). Among the two SNPs (rs10099100 (Supp. Figure 2) and rs910805 (Supp. Figure 3))

183 associated with ASD after clumping, one (rs910805) colocalized with ADHD GWAS. When

$184 p$-value was set at 1e-6, the proportion of SNPs of ASD that colocalized with ADHD GWAS

185 was $44 \%$ (7/16). Among the 18 SNPs associated with ADHD, 4 of them colocalized with

186 ASD GWAS. When $p$-value threshold was set at 1e-6, the proportion of SNPs of ASD that

187 colocalized between ADHD GWAS was 26\% (13/50). Among the 7 SNPs associated with the

188 general factor, all of them colocalized between ADHD and ASD GWAS (Figure 2 and Supp.

189 Figures 4-9). Three of them did not reach statistical significance in either the ASD and

190 ADHD GWAS: rs227378, rs2391769, and rs325506 and may be considered as new SNPs for

191 the shared genetic liability between ASD and ADHD. When the $p$-value threshold was set at

192 1e-6, the proportion of SNPs shared by ASD and ADHD as determined by Genomic SEM

193 that colocalized between ASD and ADHD GWAS was 79.2\% (19/24).

195 Functional Analyses of SNPs shared by ASD and ADHD

196 We applied FUMA to the 7 SNPs of the general factor. These 7 SNPs mapped onto 8 genes,

197 with 4 that correspond to novel genes of ASD and ADHD: MANBA, DPYD, INSM1, and 198 PAX1. 
199 Positional and eQTL mapping of deleterious coding SNPs prioritized three genes: XRN2 in 200 region 20p11.22 around rs6047319 for XRN2 (Supp. Figure 8), SORCS3 in region 10q25.1

201 around rs6584649 (Supp. Figure 9), and MANBA in region 4q24 around rs227378 (Figure

202 2). The prioritized three genes included two genes which were previously reported as 203 candidates in the study conducted on ASD by Grove et al. (24) (XRN2) and the one 204 conducted on ADHD by Demontis et al. (25) (SORCS3), while MANBA was a novel gene.

205 Gene Ontology annotations related to SORCS3 include neuropeptide receptor activity 206 (SORCS3 showed a specific expression in brain; Supp. Figure $10($ A)). Mutations in the gene 207 MANBA are associated with beta-mannosidosis, a lysosomal storage disease that has a wide 208 spectrum of neurological implications (48). Five Supplementary genes were identified by 209 chromatin interaction mapping: PTBP2 and DPYD (in region 1p21.3 around rs2391769), 210 INSM1 (in region 20p11.23 around rs6047319), and NKX2-4 and PAX1 (in region 20p11.22 211 around rs6047319) (Supp. Table 1). Among them, PTBP2 and NKX2-4 were previously 212 reported as candidates in the study conducted on ASD by Grove et al. (24) (and (49)), while 213 DPYD, INSM1, and PAX1 were novel genes. 1p21.3 microdeletions affecting DPYD have 214 been identified in individuals with ASD (50), intellectual disability (51) and has been 215 considered as a risk loci for schizophrenia $(52,53)$. INSMI is known to play a key role in 216 neurogenesis and neuroendocrine cell differentiation during embryonic and/or fetal 217 development and NKX2-4 showed a specific expression in hypothalamus (Supp. Figure 10 218 (A)). Some SNPs shared by ASD and ADHD did not map to a gene (rs 1222063, rs325506, 219 rs4916723). However, GWAS data (Supp. Data 1 for more references) suggest that the region 220 around rs325506 (5q21.2) was associated with several traits including depression (54) and 221 educational achievement (55), and that the region around rs4916723 (5q14.3) was associated 222 with neuroticism and alcohol-consumption (56,57). Globally SNPs shared by ASD and 223 ADHD were mapped to genes with specific expression in the brain (Figure 3). 


\section{Functional Analyses of SNPs Specific to ASD}

226 We applied FUMA to the SNP specific to ASD (rs10099100). Positional mapping of

227 deleterious coding SNPs prioritized two genes (SOX7 and RP1L1) which were previously

228 reported as candidates by Grove et al. (24) and Alonso-Gonzalez et al. (49). These genes

229 showed a low brain-specific expression (Figure 3 and Supp. Figure $10(B)$ ).

\section{Functional Analyses of SNPs Specific to ADHD}

231 We applied FUMA to the 14 genome-wide significant SNPs specific to ADHD. Positional 232 mapping of deleterious coding SNPs (22 genes), eQTL mapping (5 genes), and chromatin 233 interaction mapping (27 genes) prioritized 37 unique genes (some genes identified by 234 multiple mapping methods; Supp. Table 3). We used different eQTL and chromatin mapping 235 parameters on FUMA than the study by Demontis et al. (25). Therefore only 15 out of the 34 236 genes were previously reported as candidate genes by Demontis et al. (25). Each SNPs 237 rs8039398, rs28411770, and rs704067 were mapped to a single prioritized gene (respectively 238 SEMA6D, PCDH7 and DUSP6) and were therefore highly likely to drive the association 239 signal. Tissue expression of the 37 genes did not indicate a clear brain-specific expression 240 (Figure 3).

242 Bidirectional Mendelian Randomization Analysis

243 Using bidirectional Mendelian randomization, we found that the risk of ASD was associated 244 with an increased risk of ADHD across all MR approaches ( $\beta-$ MR-IVW=0.51 (0.09), $p$ 245 value $<0.001$, number of SNPs=15; Figure 4 and Supp. Table 4). Testing the reverse 246 direction, we found that the risk of ADHD was associated with an increased risk of ASD ( $\beta-$ 247 MR-IVW=0.34 (0.05), $p$-value $<0.001$, number of SNPs=41) across most MR approaches. 
248 MR-Egger implicated an effect from ASD to ADHD $\left(\beta=0.76(0.34), p\right.$-value $=0.025, \mathrm{I}_{\mathrm{GX}}$

249 statistic $=96.0 \%$ ) but no significant effect in the reverse direction (ADHD to ASD, $\beta=0.06$

$250(0.21), p$-value $=0.8, \mathrm{I}_{\mathrm{GX}}$ statistic $\left.=96.1 \%\right)$.

251 Leave-one-out sensitivity analyses did not indicate a disproportionate influence of an 252 individual SNP in any of our MR-IVW analyses. MR-Egger did not indicate directional 253 pleiotropy in both directions.

\section{DISCUSSION}

256 Using powerful multivariate genomic approaches, we examined the joint genetic architecture

257 of ASD and ADHD. To date, none of the SNPs identified as genome-wide significant in the 258 univariate GWAS of ASD and ADHD were shared between the two disorders. Implementing 259 genomic structural equation modelling, we identified 7 novel shared risk SNPs between ASD 260 and ADHD. Among them, three were not identified in either of the original univariate GWAS 261 of ASD and ADHD (rs227378, rs2391769 and rs325506). We also mapped 4 novel genes 262 (MANBA, DPYD, INSM1, and PAX1) to SNPs shared by ASD and ADHD, as well as 4 263 genes that had been already mapped to SNPs identified in either ASD or ADHD GWAS 264 (SORCS3, XRN2, PTBP2 and NKX2-4). Globally, these 8 genes revealed a specific 265 expression in the brain (Figure 3). MANBA and DPYD were mapped to novel SNPs 266 (rs227378 and rs2391769 respectively). Mutations in these two genes have previously 267 associated to neurodevelopmental disorders (48,50,51). The SNP rs2391769 was mapped to 268 DPYD but also to PTBP2 in the genomic loci 1p21. However, the SNP rs227378 was 269 mapped to a single prioritized gene (MANBA) and was highly likely to drive the association 270 signal in the genomic loci 4q24. 
272 Findings from the present study critically add to previous univariate GWAS studies not only

273 by identifying novel shared SNPs but also by identifying those suspected to be specific to one

274 or the other disorder. The pattern of findings appeared strikingly different between shared

275 and specific SNPs. Only one SNP (rs 10099100) was considered specific to ASD. This SNP

276 mapped two genes (SOX7 and RP1L1) with a low expression specificity for brain tissues

277 (SOX7 is mostly expressed in lung and RP1L1 in the retina). Similarly, genes mapped to

278 ADHD specific SNPs did not show a clear expression specific to brain tissues (Figure 3).

279 Our analyses therefore suggest that identifying genetic variants with specific effects on either

280 disorder may be less straightforward. The identification of specific genetic and environmental

281 factors of ASD and ADHD remains crucial to uncover the complex aetiology of these

282 neurodevelopmental disorders and should be the focus of further investigations (58).

284 Using colocalization analysis - a well-suited method to identify shared genetic risks between 285 two traits (29-32) - we confirmed that all the 7 SNPs identified with genomic structural 286 equation modelling were indeed shared between the two disorders. In addition, a substantial 287 part of SNPs for ASD were associated with ADHD and vice versa. We found that $44 \%$ 288 percent of the SNPs associated with ASD colocalized with ADHD SNPs, and 26\% of the 289 SNPs associated with ADHD colocalized with ASD SNPs (at $p<1 \mathrm{e}-6$ ). These results are 290 consistent with the abundant evidence for shared genetic factors in ASD and ADHD from 291 family-based $(16,17)$, twin-based $(18,19)$, and clinical studies on rare genetic variants $(20-$ 292 22). In addition, results from bidirectional Mendelian randomisation analyses indicated that 293 ASD increased the risk of ADHD and vice versa. These results suggest that ADHD leads to 294 secondary impairments in social interaction and behavioral flexibility and that ASD 295 contributes to secondary attention problems, hyperactive and impulsive behavior. 


\section{Limitations}

298 Although summary statistics for ASD and ADHD were obtained from the largest samples

299 available, they enable the identification of only a few SNPs at the conventional genome-wide

$300 p$-value threshold of 1e-8 (and thus few genes, especially for ASD). This contrasts with the

301 findings that many more genes have been linked to ASD or ADHD with regards to rare

302 genetic variants (20-22). GWAS of ASD and ADHD based on larger samples are needed to

303 explore the genetic complexity of these disorders. Yet, the results of our colocalization were

304 not affected by the number of SNPs. In fact, our estimates of the proportion of ASD

305 associated SNPs that colocalize with ADHD associated SNPs and the proportion of ADHD

306 associated SNPs that colocalize with ASD associated SNPs were very similar at the

307 conventional $p_{\text {genome-wide }}<5$ e-8 (50\% and 22\% respectively) and at $p<1 \mathrm{e}-6$ (44\% and $26 \%$,

308 respectively).

\section{Conclusion}

311 Our findings support that ADHD and ASD, two of the most common neurodevelopmental

312 disorders, share several common genetic risk variants. Our study revealed that about about

313 half of the common genetic variants associated with ASD are also linked to ADHD, and

314 about one quarter of the common genetic variants associated with ADHD are also linked to

315 ASD. Using powerful multivariate genomic approaches, we identified 7 novel SNPs shared

316 between ADHD and ASD, including three SNPs not identified in the univariate GWAS of

317 ASD and ADHD, and mapped 4 novel genes (MANBA, DPYD, INSM1, and PAX1) to SNPs

318 shared by ASD and ADHD. Further investigation of these pathways may help identify new

319 targets for treatment of these disorders. 
320

321

322

323

324

325

326

327

328

329

330

331

332

333

334

335

336

337

338

339

340

341

342

343

344

345

\section{References}

1. American Psychiatric Association (2013): Draft DSM-V Criteria for ADHD. www.adhdbasics.org/ref/DSM-V.pdf. Retrieved from www.adhdbasics.org/ref/DSMV.pdf

2. Willcutt EG (2012): The Prevalence of DSM-IV Attention-Deficit/Hyperactivity Disorder: A Meta-Analytic Review. Neurotherapeutics 9: 490-499.

3. Baio J (2018): Prevalence of Autism Spectrum Disorder Among Children Aged 8 Years Autism and Developmental Disabilities Monitoring Network, 11 Sites, United States, 2014. MMWR Surveill Summ 67. https://doi.org/10.15585/mmwr.ss6706a1

4. Lord C, Brugha TS, Charman T, Cusack J, Dumas G, Frazier T, et al. (2020): Autism spectrum disorder. Nat Rev Dis Primer 6: 1-23.

5. Santosh PJ, Mijovic A (2004): Social impairment in Hyperkinetic Disorder - relationship to psychopathology and environmental stressors. Eur Child Adolesc Psychiatry 13: $141-150$.

6. Clark T, Feehan C, Tinline C, Vostanis P (1999): Autistic symptoms in children with attention deficit-hyperactivity disorder. Eur Child Adolesc Psychiatry 8: 50-55.

7. Hollingdale J, Woodhouse E, Young S, Fridman A, Mandy W (2019): Autistic spectrum disorder symptoms in children and adolescents with attention-deficit/hyperactivity disorder: a meta-analytical review. Psychol Med 1-14.

8. Leyfer OT, Folstein SE, Bacalman S, Davis NO, Dinh E, Morgan J, et al. (2006):

Comorbid psychiatric disorders in children with autism: interview development and rates of disorders. J Autism Dev Disord 36: 849-861.

9. Lai M-C, Kassee C, Besney R, Bonato S, Hull L, Mandy W, et al. (2019): Prevalence of co-occurring mental health diagnoses in the autism population: a systematic review and meta-analysis. Lancet Psychiatry 6: 819-829. 
346 10. Cross-Disorder Group of the Psychiatric Genomics Consortium, Lee SH, Ripke S, Neale

347 BM, Faraone SV, Purcell SM, et al. (2013): Genetic relationship between five

348 psychiatric disorders estimated from genome-wide SNPs. Nat Genet 45: 984-994.

349 11. Sullivan PF, Daly MJ, O’Donovan M (2012): Genetic architectures of psychiatric

350 disorders: the emerging picture and its implications. Nat Rev Genet 13: 537-551.

351 12. Bhutta AT, Cleves MA, Casey PH, Cradock MM, Anand KJS (2002): Cognitive and

352 behavioral outcomes of school-aged children who were born preterm: a meta-analysis.

$353 \quad$ JAMA 288: 728-737.

354 13. Agrawal S, Rao SC, Bulsara MK, Patole SK (2018): Prevalence of Autism Spectrum

355 Disorder in Preterm Infants: A Meta-analysis. Pediatrics 142.

356 https://doi.org/10.1542/peds.2018-0134

357 14. Christensen J, Pedersen L, Sun Y, Dreier JW, Brikell I, Dalsgaard S (2019): Association

358 of Prenatal Exposure to Valproate and Other Antiepileptic Drugs With Risk for

359 Attention-Deficit/Hyperactivity Disorder in Offspring. JAMA Netw Open 2: e186606.

360 15. Stadelmaier R, Nasri H, Deutsch CK, Bauman M, Hunt A, Stodgell C, et al. (2017):

361 Exposure to sodium valproate during pregnancy: Facial features and signs of autism.

362 Birth Defects Res 109: 1134-1143.

363 16. Ghirardi L, Brikell I, Kuja-Halkola R, Freitag CM, Franke B, Asherson P, et al. (2018):

364 The familial co-aggregation of ASD and ADHD: a register-based cohort study. Mol

$365 \quad$ Psychiatry 23: 257-262.

366 17. Septier M, Peyre H, Amsellem F, Beggiato A, Maruani A, Poumeyreau M, et al. (2019):

367 Increased risk of ADHD in families with ASD. Eur Child Adolesc Psychiatry 28:

$368 \quad 281-288$. 
369 18. Constantino JN, Hudziak JJ, Todd RD (2003): Deficits in reciprocal social behavior in

370 male twins: evidence for a genetically independent domain of psychopathology. $J$ Am

$371 \quad$ Acad Child Adolesc Psychiatry 42: 458-467.

372 19. Ronald A, Simonoff E, Kuntsi J, Asherson P, Plomin R (2008): Evidence for overlapping

373 genetic influences on autistic and ADHD behaviours in a community twin sample. $J$

$374 \quad$ Child Psychol Psychiatry 49: 535-542.

375 20. Satterstrom FK, Walters RK, Singh T, Wigdor EM, Lescai F, Demontis D, et al. (2019):

376 Autism spectrum disorder and attention deficit hyperactivity disorder have a similar

377 burden of rare protein-truncating variants. Nat Neurosci 22: 1961-1965.

378 21. Martin J, Cooper M, Hamshere ML, Pocklington A, Scherer SW, Kent L, et al. (2014):

379 Biological overlap of attention-deficit/hyperactivity disorder and autism spectrum

380 disorder: evidence from copy number variants. J Am Acad Child Adolesc Psychiatry

$381 \quad$ 53: 761-770.e26.

382 22. Gudmundsson OO, Walters GB, Ingason A, Johansson S, Zayats T, Athanasiu L, et al.

383 (2019): Attention-deficit hyperactivity disorder shares copy number variant risk with

384 schizophrenia and autism spectrum disorder. Transl Psychiatry 9: 1-9.

385 23. Gaugler T, Klei L, Sanders SJ, Bodea CA, Goldberg AP, Lee AB, et al. (2014): Most

386 genetic risk for autism resides with common variation. Nat Genet 46: 881-885.

387 24. Grove J, Ripke S, Als TD, Mattheisen M, Walters RK, Won H, et al. (2019):

388 Identification of common genetic risk variants for autism spectrum disorder. Nat

$389 \quad$ Genet 51: 431-444.

390 25. Demontis D, Walters RK, Martin J, Mattheisen M, Als TD, Agerbo E, et al. (2019):

391 Discovery of the first genome-wide significant risk loci for attention

392 deficit/hyperactivity disorder. Nat Genet 51: 63-75. 
393 26. Grotzinger AD, Rhemtulla M, de Vlaming R, Ritchie SJ, Mallard TT, Hill WD, et al.

394 (2019): Genomic structural equation modelling provides insights into the multivariate 395 genetic architecture of complex traits. Nat Hum Behav 3: 513-525.

396 27. Giambartolomei C, Zhenli Liu J, Zhang W, Hauberg M, Shi H, Boocock J, et al. (2018):

397 A Bayesian framework for multiple trait colocalization from summary association $398 \quad$ statistics. Bioinforma Oxf Engl 34: 2538-2545.

399 28. Amare AT, Vaez A, Hsu Y-H, Direk N, Kamali Z, Howard DM, et al. (2019): Bivariate

400 genome-wide association analyses of the broad depression phenotype combined with

401 major depressive disorder, bipolar disorder or schizophrenia reveal eight novel

402 genetic loci for depression. Mol Psychiatry. https://doi.org/10.1038/s41380-018-0336-

4036

404 29. Fortune MD, Guo H, Burren O, Schofield E, Walker NM, Ban M, et al. (2015): Statistical 405 colocalization of genetic risk variants for related autoimmune diseases in the context 406 of common controls. Nat Genet 47: 839-846.

407 30. Siewert KM, Voight BF (2018): Bivariate Genome-Wide Association Scan Identifies 6

408 Novel Loci Associated With Lipid Levels and Coronary Artery Disease. Circ

$409 \quad$ Genomic Precis Med 11: e002239.

410 31. Dobbyn A, Huckins LM, Boocock J, Sloofman LG, Glicksberg BS, Giambartolomei C, et 411 al. (2018): Landscape of Conditional eQTL in Dorsolateral Prefrontal Cortex and Co412 localization with Schizophrenia GWAS. Am J Hum Genet 102: 1169-1184.

413 32. Bhalala OG, Nath AP, UK Brain Expression Consortium, Inouye M, Sibley CR (2018):

414 Identification of expression quantitative trait loci associated with schizophrenia and $415 \quad$ affective disorders in normal brain tissue. PLoS Genet 14: e1007607.

416 33. Taylor MJ, Charman T, Robinson EB, Plomin R, Happé F, Asherson P, Ronald A (2013):

417 Developmental associations between traits of autism spectrum disorder and attention 
418 deficit hyperactivity disorder: a genetically informative, longitudinal twin study.

$419 \quad$ Psychol Med 43: 1735-1746.

420 34. Pingault J-B, O’Reilly PF, Schoeler T, Ploubidis GB, Rijsdijk F, Dudbridge F (2018):

421 Using genetic data to strengthen causal inference in observational research. Nat Rev

$422 \quad$ Genet 19: 566-580.

423 35. Soler Artigas M, Sánchez-Mora C, Rovira P, Richarte V, Garcia-Martínez I, Pagerols M, 424 et al. (2019): Attention-deficit/hyperactivity disorder and lifetime cannabis use:

425 genetic overlap and causality. Mol Psychiatry. https://doi.org/10.1038/s41380-018-

$426 \quad 0339-3$

427 36. Nivard M (2019): MichelNivard/GenomicSEM: Structural equation modeling based on 428 GWAS summary statistics version 0.0.2 from GitHub. Retrieved December 14, 2019, 429 from https://rdrr.io/github/MichelNivard/GenomicSEM/

430 37. Wallace C, Giambartolomei C, Plagnol V (2019): Coloc: Colocalisation Tests of Two

431 Genetic Traits, version 3.2-1. Retrieved December 14, 2019, from https://CRAN.R-

$432 \quad$ project.org/package $=$ coloc

433 38. Foley C, Staley J (2019): hyprcoloc: HyPrColoc in jrs95/hyprcoloc: Hypothesis

434 Prioritisation in multi-trait Colocalisation (HyPrColoc) analyses. Retrieved December

$435 \quad 14,2019$, from https://rdrr.io/github/jrs95/hyprcoloc/man/hyprcoloc.html

436 39. Giambartolomei C, Vukcevic D, Schadt EE, Franke L, Hingorani AD, Wallace C, Plagnol

$437 \quad$ V (2014): Bayesian test for colocalisation between pairs of genetic association studies

$438 \quad$ using summary statistics. PLoS Genet 10: e1004383.

439 40. Wallace C (2019): Eliciting priors and relaxing the single causal variant assumption in

$440 \quad$ colocalisation analyses. bioRxiv 838946.

441 41. Watanabe K, Taskesen E, Bochoven A van, Posthuma D (2017): Functional mapping and 442 annotation of genetic associations with FUMA. Nat Commun 8: 1-11. 
443 42. Giusti-Rodriguez PM, Sullivan PF (2019): Using three-dimensional regulatory chromatin

444 interactions from adult and fetal cortex to interpret genetic results for psychiatric

445 disorders and cognitive traits. bioRxiv 406330.

446 43. Pingault J-B, Cecil C, Murray J, Munafo M, Viding E (2016): Causal inference in

447 psychopathology: A systematic review of Mendelian randomisation studies aiming to

448 identify environmental risk factors for psychopathology.

449 https://doi.org/10.17863/CAM.4366

450 44. Davies NM, Holmes MV, Smith GD (2018): Reading Mendelian randomisation studies: a 451 guide, glossary, and checklist for clinicians. BMJ 362.

$452 \quad$ https://doi.org/10.1136/bmj.k601

453 45. Burgess S, Thompson SG (2017): Interpreting findings from Mendelian randomization

$454 \quad$ using the MR-Egger method. Eur J Epidemiol 32: 377-389.

455 46. Hemani G, Bowden J, Davey Smith G (2018): Evaluating the potential role of pleiotropy

456 in Mendelian randomization studies. Hum Mol Genet 27: R195-R208.

457 47. Zhao Q, Wang J, Hemani G, Bowden J, Small DS (2019): Statistical inference in two-

458 sample summary-data Mendelian randomization using robust adjusted profile score.

459 ArXiv180109652 Math Stat. Retrieved January 15, 2020, from

460 http://arxiv.org/abs/1801.09652

461 48. Sabourdy F, Labauge P, Stensland HMFR, Nieto M, Garcés VL, Renard D, et al. (2009):

462 A MANBA mutation resulting in residual beta-mannosidase activity associated with

463 severe leukoencephalopathy: a possible pseudodeficiency variant. BMC Med Genet

$464 \quad 10: 84$.

465 49. Alonso-Gonzalez A, Calaza M, Rodriguez-Fontenla C, Carracedo A (2019): Novel Gene-

466 Based Analysis of ASD GWAS: Insight Into the Biological Role of Associated

467 Genes. Front Genet 10. https://doi.org/10.3389/fgene.2019.00733 
468 50. Carter MT, Nikkel SM, Fernandez BA, Marshall CR, Noor A, Lionel AC, et al. (2011):

Hemizygous deletions on chromosome 1p21.3 involving the DPYD gene in individuals with autism spectrum disorder. Clin Genet 80: 435-443.

51. Willemsen MH, Vallès A, Kirkels LAMH, Mastebroek M, Olde Loohuis N, Kos A, et al. (2011): Chromosome 1p21.3 microdeletions comprising DPYD and MIR137 are

52. Hill MJ, Donocik JG, Nuamah RA, Mein CA, Sainz-Fuertes R, Bray NJ (2014): Transcriptional consequences of schizophrenia candidate miR-137 manipulation in human neural progenitor cells. Schizophr Res 153: 225-230.

53. Xu B, Ionita-Laza I, Roos JL, Boone B, Woodrick S, Sun Y, et al. (2012): De novo gene mutations highlight patterns of genetic and neural complexity in schizophrenia. Nat Genet 44: 1365-1369. 
bioRxiv preprint doi: https://doi.org/10.1101/2020.04.22.054825; this version posted April 24, 2020. The copyright holder for this preprint (which was not certified by peer review) is the author/funder. All rights reserved. No reuse allowed without permission.

493 58. Uher R, Zwicker A (2017): Etiology in psychiatry: embracing the reality of poly-gene-

494 environmental causation of mental illness. World Psychiatry 16: 121-129.

495 
Table 1. Colocalization analysis of SNPs of ASD, ADHD and the general factor (using COLOC and Hyprcoloc).

\begin{tabular}{|c|c|c|c|c|c|c|c|}
\hline & \multirow[b]{2}{*}{ SNP } & \multirow[b]{2}{*}{$\mathrm{A} 1 / \mathrm{A} 2$} & \multirow[b]{2}{*}{ Chromosome } & \multirow[b]{2}{*}{$\begin{array}{l}\text { Minor allele } \\
\text { frequency }\end{array}$} & \multicolumn{3}{|c|}{ Colocalization analysis } \\
\hline & & & & & $\begin{array}{l}\text { Probability H4 } \\
\text { using COLOC }\end{array}$ & $\begin{array}{c}\text { Probability H4 } \\
\text { using } \\
\text { Hyprcoloc }\end{array}$ & $\begin{array}{c}\text { Colocalization } \\
\text { between ASD } \\
\text { and ADHD } \\
\text { GWAS }\end{array}$ \\
\hline \multirow[t]{2}{*}{ ASD GWAS } & rs10099100 & $\mathrm{C} / \mathrm{G}$ & 8 & 0.34 & 0.01 & $<0.5$ & No \\
\hline & rs910805 & $\mathrm{A} / \mathrm{G}$ & 20 & 0.24 & 0.51 & $<0.5$ & Yes $^{d}$ \\
\hline \multicolumn{8}{|l|}{ ADHD GWAS } \\
\hline & rs10261780 & $\mathrm{T} / \mathrm{C}$ & 7 & 0.43 & 0.15 & $<0.5$ & No \\
\hline & $\mathrm{rs} 118041269$ & $\mathrm{~A} / \mathrm{G}$ & 8 & 0.06 & 0.04 & $<0.5$ & No \\
\hline & rs $1222063^{\#}$ & $\mathrm{~A} / \mathrm{G}$ & 1 & 0.37 & 0.98 & 0.99 & Yes \\
\hline & rs12265655 & $\mathrm{T} / \mathrm{C}$ & 10 & 0.23 & 0.89 & 0.90 & Yes \\
\hline & rs212178 & $\mathrm{A} / \mathrm{G}$ & 16 & 0.10 & 0.51 & $<0.5$ & Yes $^{d}$ \\
\hline & $\mathrm{rs} 2275180$ & $\mathrm{~A} / \mathrm{G}$ & 1 & 0.36 & 0.01 & $<0.5$ & No \\
\hline & rs 28411770 & $\mathrm{~T} / \mathrm{C}$ & 4 & 0.38 & 0.09 & $<0.5$ & No \\
\hline & rs 304132 & $\mathrm{~A} / \mathrm{G}$ & 5 & 0.40 & 0.03 & $<0.5$ & No \\
\hline & rs 37452 & $\mathrm{~A} / \mathrm{C}$ & 1 & 0.32 & 0.01 & $<0.5$ & No \\
\hline & rs 4858241 & $\mathrm{~T} / \mathrm{G}$ & 3 & 0.33 & 0.04 & $<0.5$ & No \\
\hline & rs4916723" & $\mathrm{A} / \mathrm{C}$ & 5 & 0.41 & 0.86 & 0.86 & Yes \\
\hline & rs704067 & $\mathrm{A} / \mathrm{G}$ & 12 & 0.43 & 0.17 & $<0.5$ & No \\
\hline & rs76013678 & $\mathrm{A} / \mathrm{C}$ & 8 & 0.06 & 0.04 & $<0.5$ & No \\
\hline & rs8039398 & $\mathrm{T} / \mathrm{C}$ & 15 & 0.46 & 0.02 & $<0.5$ & No \\
\hline & rs 72678674 & $\mathrm{~A} / \mathrm{G}$ & 1 & 0.25 & 0.01 & $<0.5$ & No \\
\hline & rs34898652 & $\mathrm{A} / \mathrm{G}$ & 1 & 0.36 & 0.01 & $<0.5$ & No \\
\hline & $\mathrm{rs} 113551349$ & $\mathrm{~T} / \mathrm{C}$ & 1 & 0.28 & 0.01 & $<0.5$ & No \\
\hline & rs10890255 & $\mathrm{A} / \mathrm{G}$ & 1 & 0.26 & 0.01 & $<0.5$ & No \\
\hline \multicolumn{8}{|c|}{ General factor GWAS } \\
\hline & $\mathrm{rs} 1222063^{\#}$ & $\mathrm{~A} / \mathrm{G}$ & 1 & 0.37 & 0.98 & 0.99 & Yes \\
\hline & rs 227378 & $\mathrm{~A} / \mathrm{C}$ & 4 & 0.34 & 0.90 & 0.93 & Yes \\
\hline & rs 2391769 & $\mathrm{~A} / \mathrm{G}$ & 1 & 0.33 & 0.89 & 0.93 & Yes \\
\hline & rs325506 & $\mathrm{C} / \mathrm{G}$ & 5 & 0.44 & 0.73 & 0.84 & Yes \\
\hline & rs $4916723^{\#}$ & $\mathrm{~A} / \mathrm{C}$ & 5 & 0.41 & 0.86 & 0.86 & Yes \\
\hline & rs6047319 & $\mathrm{A} / \mathrm{G}$ & 20 & 0.33 & 0.51 & 0.51 & Yes \\
\hline & rs6584649 & $\mathrm{T} / \mathrm{G}$ & 10 & 0.23 & 0.89 & 0.90 & Yes \\
\hline
\end{tabular}

Yes ${ }^{\mathrm{d}}$ Discordance between COLOC and Hyprcoloc.

\# Two of the 7 SNPs (rs1222063 and rs4916723) of the general factor GWAS had been found in ADHD GWAS. 
bioRxiv preprint doi: https://doi.org/10.1101/2020.04.22.054825; this version posted April 24, 2020. The copyright holder for this preprint (which was not certified by peer review) is the author/funder. All rights reserved. No reuse allowed without permission.

Figure 1. Manhattan plot of the general factor of ASD and ADHD using Genomic SEM.

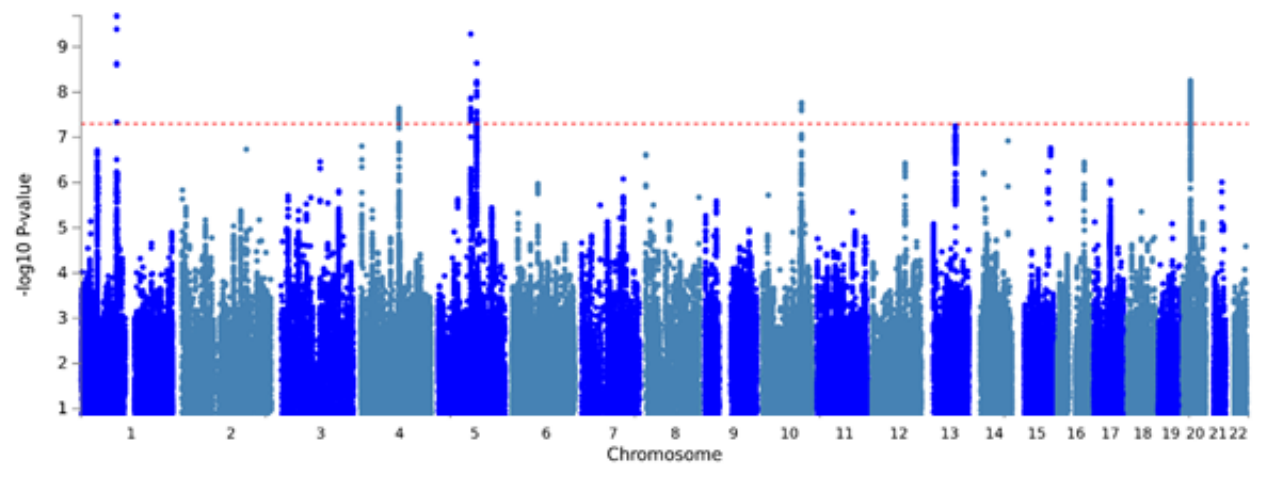


Figure 2. Region around rs227378 (250kb around the SNP) in ADHD (upper figure [P1]) and ASD (lower figure [P2]) GWAS.
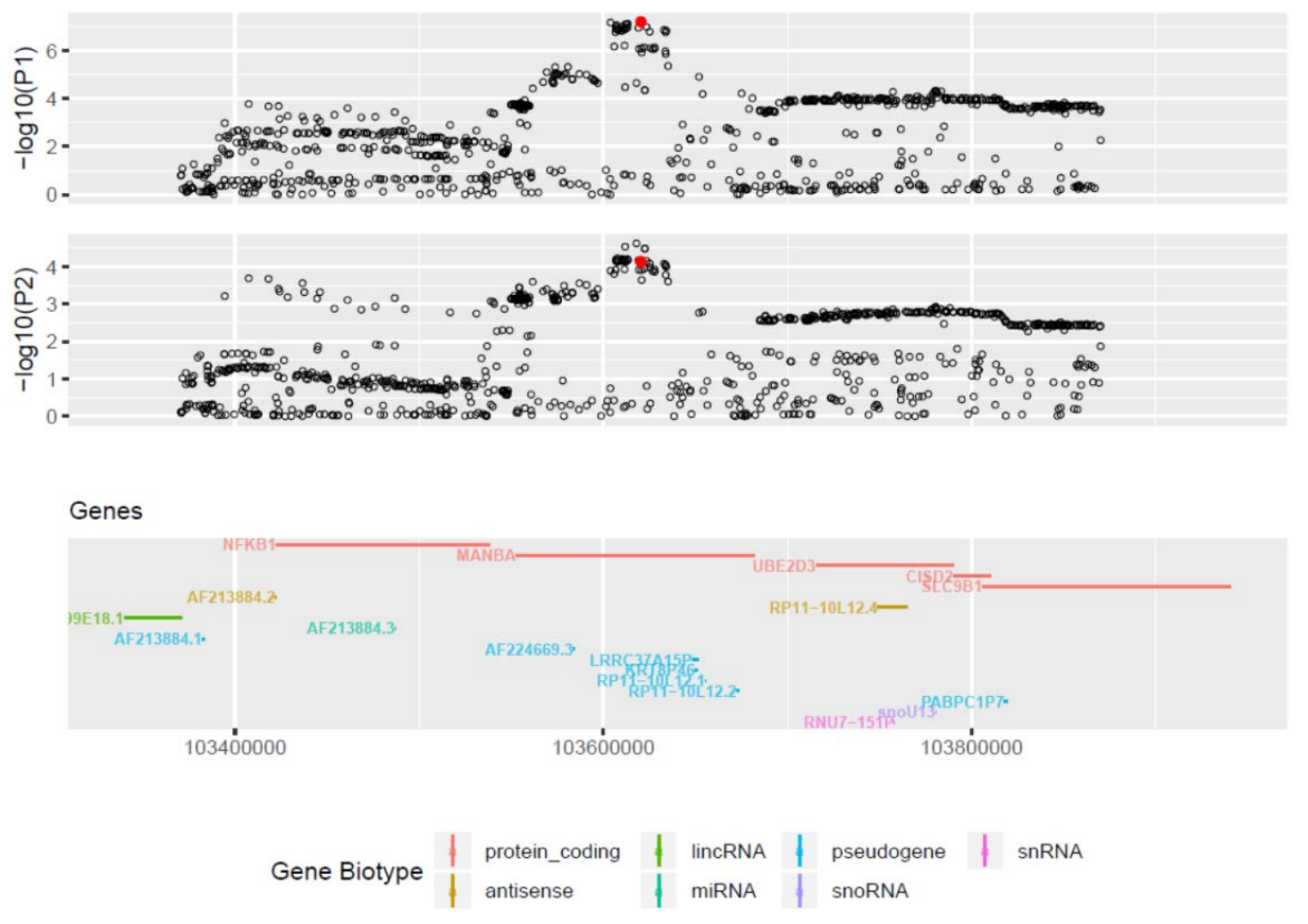
Figure 3. Tissue specific expression patterns (average of normalized expression per label) using GTEx v6 RNA-seq data (FUMA GENE2FUNC) of the SNPs shared by ASD and ADHD and those that are specific to ASD and ADHD.

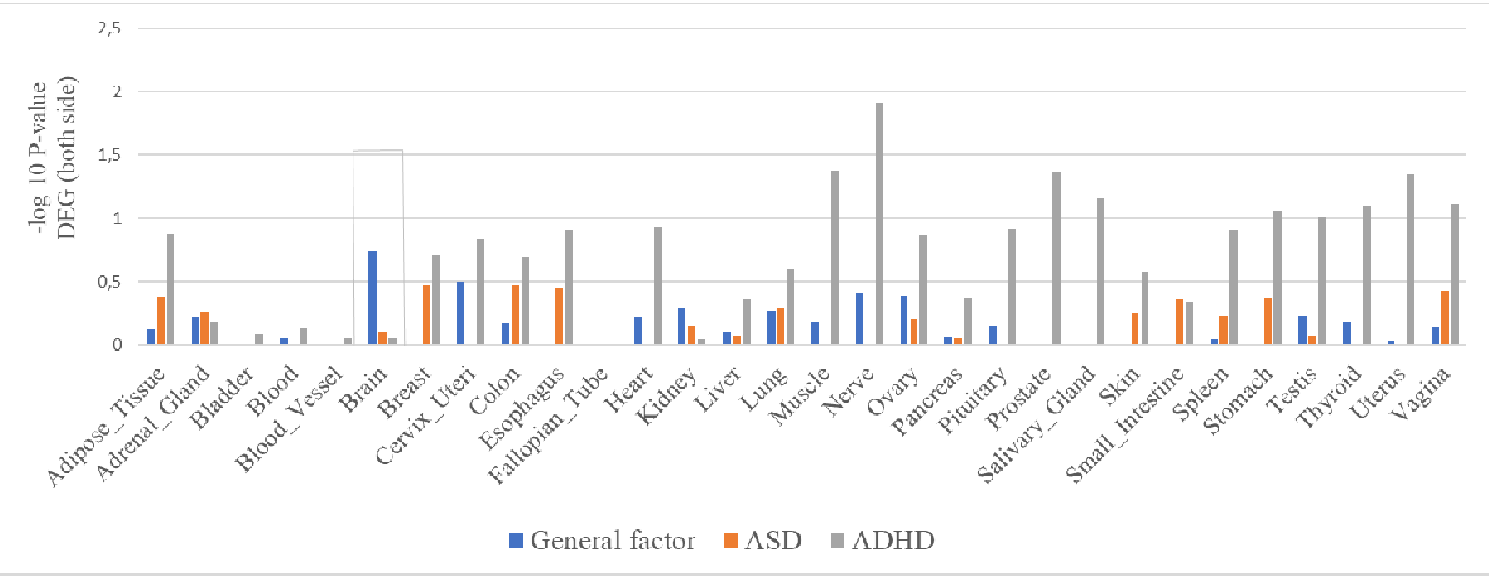


Figure 4. Bidirectional MR using the inverse weighted variance (IVW) estimator. Causal relationship between ASD as exposure ( $p$-value at 1e-6) and ADHD as outcome (left). Causal relationship between ADHD as exposure ( $p$-value at 1e-6) and ASD as outcome (right).
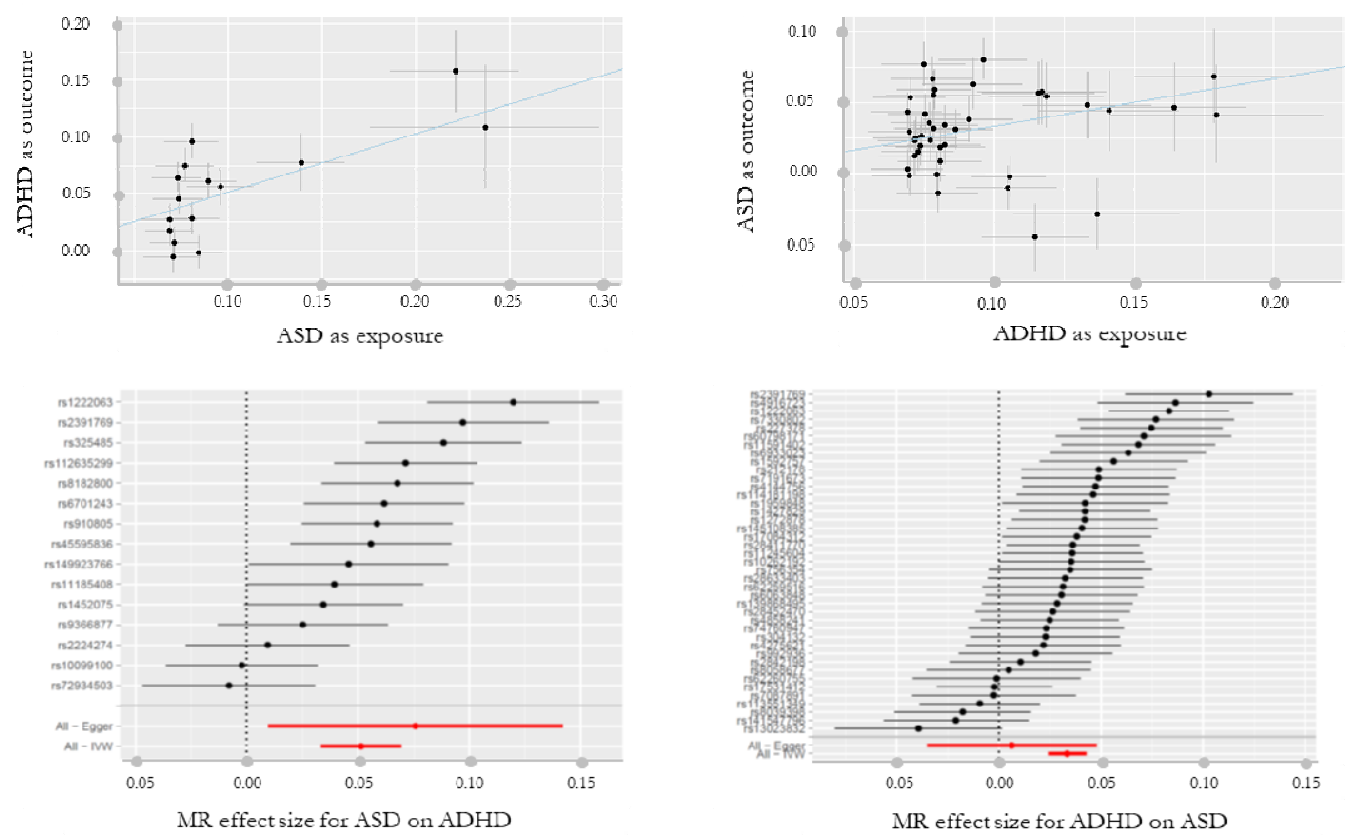